\title{
Scaffold electrodes based on Thioctic acid-capped gold nanoparticles coordinated Alcohol Dehydrogenase and azure A films for high performance biosensor.
}

C. Gómez-Anquela a, T. García-Mendiola a , José M. Abad ${ }^{\text {a }}$, M. Pita ${ }^{c}$, F. Pariente ${ }^{\text {a }}$, E. Lorenzo ${ }^{\mathrm{a}, \mathrm{b} *}$

${ }^{a}$ Department of Analytical Chemistry, Universidad Autónoma de Madrid, 28049

b IMDEA Nanociencia, Madrid, Spain.

c Instituto de Catalisis y Petroleoquimica, CSIC. C/ Marie Curie, 2, L10. 28049 Madrid, Spain.

cesar.anquela@uam.es, tania.garcia@uam.es, josemaria.abad@uam.es, marcospita@ic p.csic.es, felix.pariente@uam.es

*Corresponding author. Tel.: +34 91497 4488; fax: +34 914974931.

E-mail address: encarnacion.lorenzo@uam.es (E.Lorenzo)

Postal address: Facultad de Ciencias. c/ Francisco Tomás y Valiente, 7. Departamento de Química Analítica y Análisis Instrumental. Universidad Autónoma de Madrid, 28049, Madrid, Spain.

\begin{abstract}
Nanometric size gold nanoparticles capped with thiotic acid are used to coordinate with the Zn (II) present in the catalytic center of Alcohol Dehydrogenase (ADH). In combination with the NADH oxidation molecular catalyst Azure A, electrografted onto carbon screen-printed electrodes, they are used as scaffold electrodes for the construction of a very efficient ethanol biosensor. The final biosensing device exhibits a highly efficient ethanol oxidation with low overpotential of $-0.25 \mathrm{~V}$ besides a very good analytical performance with a detection limit of $0.14 \pm 0.01 \mu \mathrm{M}$ and a stable response for more than one month.
\end{abstract}


Keywords: Alcohol dehydrogenase, Biosensor, Gold nanoparticles, electrografting.

\section{Introduction}

Accurate and sensitive determination of ethanol in different samples is an important challenge in many diverse areas. In particular, in clinical and forensic chemistry the toxic effects caused by the presence of high levels of alcohol in blood result in several disorders that can lead to severe health damages, besides work and traffic accidents and generally loss of quality of life. On the other hand, in fermentation processes of wine, beer or in distillation of spirits control of ethanol concentration is essential to prevent damage in the process. Moreover, recently there has been an extraordinary interest, by the chemical industry, in the production of ethanol as an alternative fuel to other petroleum products. Thus, several analytical methodologies have been developed to determine ethanol either in biological samples, such as blood, saliva, urine, etc. or in samples from crude extracts obtained from a determined fermentation process. These methodologies among others include spectrophotometry (Barzana et al. 1989), high performance liquid chromatography (Li et al. 2000), gas chromatography (Ragazzo-Sanchez et al. 2005; Wanekaya et al. 2005) or refractometry (Lazarova et al. 1987). Many of these methods provide accurate and precise determination of ethanol but present some disadvantages derived from large and tedious protocols of sample preparation that results in large analysis times. Moreover, most of these methodologies require an extensive an expensive instrumentation.

The importance of ethanol as analyte and the growing interest of determining the alcohol levels in situ, outside the laboratory, demand for new methods of analysis leading to portable devices capable to determine it in whole samples (Zhen et al. 2011) in a fast, simple, and accurate form. Biosensors based on selective ethanol conversion enzymes, are a valuable alternative methodology for sensitive, accurate, selective, inexpensive and rapid determination of alcohol concentration in all types of samples. In this way, two kinds of enzymes, Alcohol Dehydrogenase (ADH) (Tkac et al. 2009; Wiseman 1988) and Alcohol Oxidase (AOx) have been used in the fabrication of alcohol biosensors (Goswami et al. 2013). In particular, the first one has been most widely used due to its high stability, which makes it more suitable to resist the immobilization process. 
Yeast Alcohol Dehydrogenase I (ADH) belongs to a group of redox enzymes that are present in many organisms. This group of enzymes catalyzes the oxidation of alcohols to their corresponding aldehydes or ketones. The oxidized form of nicotinamide adenine dinucleotide cofactor $\left(\mathrm{NAD}^{+}\right)$takes the electronic pair involved in the process and is transformed into the corresponding reduced form (NADH). In this case the cofactor acts as a second substrate, for this reason these enzymes are called dehydrogenases dependent on $\mathrm{NAD}^{+}$. Yeast alcohol dehydrogenase $(\mathrm{ADH})$ is a tetramer of four identical subunits (Raj et al. 2014) with 347 amino acid residues each and a calculated mass of 147.396 Da (36.849 Da/subunit), each subunit contains two zinc (II) ions that are tetracoordinated (Rubach and Plapp 2003). The zinc ion located in the catalytic lobe is coordinated with two cysteines, one glutamic and one histidine. It is involved in the catalytic oxidation of ethanol. The other zinc ion is coordinated with four cysteines (Raj et al. 2014) and is located in a structural lobe. In the active center this enzyme has two binding domains: the first one binds the substrate (ethanol) and the second one is reserved for the oxidized form of the cofactor (Hammes-Schiffer and Benkovic 2006). Electrochemical biosensors based on yeast ADH have the advantage of using a stable protein (compared to biosensors based on AOx) but also have the disadvantage of being dependent on the presence of $\mathrm{NAD}^{+}$into the biocatalytic layer. To avoid this inconvenience, the cofactor should be added to the solution or immobilized along with the enzyme on the electrode surface. In addition, the reduced form of this cofactor (NADH) needs large overpotential for reoxidation (Bartlett et al. 2002; Lee and Tsai 2009; Rubianes and Rivas 2005), which increases the risk of interferences from substances present in the matrix. The large overvoltage intrinsic with the NADH oxidation onto solid electrodes is due to the formation of no electroactive oxidation products that rapidly adsorb on the electrode surface fouling it (Bartlett et al. 2002; Lee and Tsai 2009). To overcome this problem, the electrode surface can be modified with substances capable to act as redox mediators reducing the overpotential (García Mullor et al. 1996; Pariente et al. 1995) Other approaches to prevent the electrode fouling are based on the modification of electrode surface with carbon nanotubes or graphene sheets (Banks and Compton 2005; Banks et al. 2005). These studies have demonstrated that carbon nanotubes show electrocatalytic activity towards NADH oxidation by themselves or by their antifouling capabilities (Banks et al. 2005; Lee and Tsai 2009). The problem with these nanostructured electrodes is the poor 
solubility of the starting nanocarbon sources, which involve large protocols of suspension-solubilization and the lack of precise analytical tests to quantify the real electroactive area of the modified electrode.

Recently, we have modified screen-printed carbon electrodes (SPCE) with Azure A (AA) by electro-grafting of the corresponding diazonium salt generated in situ. Such modified electrodes show a potent and persistent electrocatalytic effect to the oxidation of NADH (Revenga-Parra et al. 2012). Moreover, it has been reported that electrodes modified with phenothiazine dyes show a strong affinity for proteins and DNA (Huang and Wang 2010). The binding strength have been estimated to be around $200 \mathrm{nN}$, equivalent to $10^{2} \mathrm{C}-\mathrm{C}$ single bonds (Wu et al. 2008), suggesting that phenothiazine modified electrodes can be used in the construction of biosensor. In particular, we thought that AA modified SPEs can be a good choice for the development of dehydrogenases based biosensors for two reasons: i) the phenothiazine modified surface can act as an effective molecular adhesive for the enzyme and ii) it can be an efficient redox mediator for electroxidation of the NADH enzymatically generated. However, it is well known that the electronic coupling between the enzyme and the electrode is relatively weak. To overcome this problem, nanoparticle-enzyme hybrid systems has been employed (Katz and Willner 2004; Niemeyer 2001; Scott et al. 2008; Willner et al. 2007a; Gutierrez-Sanchez 2012). Most of these approaches are based on a simple deposition of nanoparticles and enzymes on an electrode support (Jia et al. 2002; Zhao et al. 1996). In general, large nanoparticles have been used, and the enzyme has been immobilized on the nanoparticles used as an extension of the electrode surface (Jensen et al. 2007; Jia et al. 2002). However, the incorporation of a nanoparticle into the protein offers an interesting route to improve electron transfer. In this case, a suitable nanoparticle must be employed. For instance, previous works have demonstrated that the presence of a small $(1.4 \mathrm{~nm})$ gold nanoparticle can enhance exceptionally the turnover rate of metaloenzymes based bioelectrocatalysis by optimizing electrical connectivity (Abad et al. 2009; Xiao et al. 2003).

Chemical derivatization of gold nanoparticles or direct coupling to biomolecules is further facilitated by the use of capping ligands containing, in addition to the thiol group, a functional group such as an amine or a carboxylic moiety that can be used for linking to proteins through either covalent or noncovalent interactions. In this sense, thioctic acid-capped nanoparticles show high stability in aqueous solutions and serve 
well as a scaffold for direct coupling of proteins retaining their biological function (Abad et al. 2005).

In the present work we have applied the concept to achieve both specific recognition of a metallic center and enhancement of the electrical connectivity to an electrode by using the coordination properties of the metal center of the enzyme for its specific recognition by ligands attached to a gold nanoparticle. The interconnection strategy followed is based on the introduction of a carboxylate-functionalized gold nanoparticle of suitable size inside the pocket of the enzyme to achieve direct coordination with its metal. Following this strategy we describe the construction and characterization of an improved ethanol biosensor based on the direct immobilization of alcohol deshydrogenase coordinated through the catalytic zinc (II) to thioctic acid capped gold nanoparticles, on the surface of an electro-grafted phenothiazine (AA) layer which have a dual function: bioadhesive and NADH oxidative catalyst. The prevention of enzyme leakage from the biosensing layer is also studied. 


\section{Experimental section}

\subsection{Chemicals and stock solutions}

Azure A (AA), $\beta$-Nicotinamide adenine dinucleotide disodium salt hydrate, Alcohol dehydrogenase $(\mathrm{ADH})$ from Saccharomyces Cerevisiae $(\geq 300 \mathrm{U} / \mathrm{mg}$; EC 1.1.1.1), Thioctic acid (TO), Sodium tetrachloroaurate (III) hydrate $\left(\mathrm{HAuCl}_{4}\right)$, Tetraoctylammonium bromide (TOABr), Sodium borohydride $\left(\mathrm{NaBH}_{4}\right)$, polyethilenglycol (PEG), nafion, polyethylenimine (PEI) and Chitosan medium molecular weight (CHIT) were purchased from Sigma Chemical Co and used as received. Acetic acid solution was obtained from Fluka. Sodium nitrite was purchased from Riedel-de-Haën and Absolute ethanol (EtOH) was obtained from Fischer Scientific. Other reagents used in this work were of analytical grade.

Stock solutions of ADH were prepared in $0.1 \mathrm{M} \mathrm{pH} 8.0$ phosphate buffer solution (PBS) at a final concentration of $10.0 \mathrm{mg} / \mathrm{mL}(8.3 \mathrm{U} / \mu \mathrm{L})$ and stored frozen at $-20^{\circ} \mathrm{C}$. In these conditions the enzyme activity remains stable at least four weeks.

Ultrapure grade water $(18.2 \mathrm{M} \Omega \mathrm{cm})$ from a Millipore Milli-Q system was used in the experiments.

\subsection{Apparatus}

Electrochemical experiments were carried out at room temperature using an Autolab PGSTAT 30 potentiostat from Eco-Chemie (KM Utrecht, The Netherlands) using the software package GPES 4.9 (General Purpose Elec. Experiments) and were performed in a homemade single compartment electrochemical cell. Integrated screen-printed carbon electrodes (4 mm diameter, SPCEs) from DropSens S.L (Oviedo, Spain) including a silver pseudoreference electrode and a carbon counter electrode were used. The electrodes were connected using a SPE connector (DropSens S. L.) as interface. $\mathrm{O}_{2}$-free nitrogen was used to remove the oxygen from the solutions and a continuous flow of nitrogen was maintained during the voltammetric experiments.

Total reflection x-ray fluorescence (TXRF) analysis was performed by using a TXRF Bruker PicoFox S2 spectrometer (Bruker AXS Microanalysis GmbH, Berlin, Germany) equipped with a X-ray tube which included a Mo anode, a multilayer monochromator and a Si (SDD) detector with an active area of $30 \mathrm{~mm}^{2}$ were used.

Transmission electron microscopy (TEM) measurements were carried out with a 200 KV JEOL 2100 TEM/STEM microscope. Coupled Energy-dispersive X-ray analyzer (EDX) from Oxford Instruments was used for the elemental surface analysis. High 
angular annular dark field scanning transmission electron microscopy (HAADF-STEM) was also used to collect z-contrast images.

UV-Vis spectra were recorded in a double beam PharmaSpec UV-1700 series (Shimadzu Co., Kyoto, Japan) by using a $1 \mathrm{~cm}$ optical path length quartz cells.

\subsection{Procedures}

Synthesis and characterization of functionalized gold nanoparticles.

Thioctic acid capped gold nanoparticles (TO-AuNPs) were prepared by the twophase method described by Brust (Brust et al. 1994) (more detail in supporting information). TEM was employed to estimate the size of the as-prepared TO-AuNPs (Figure 1S). The average core diameter was found to be around $3.4 \pm 0.8 \mathrm{~nm}$ (Figure 1 of SI). This value is too small so they do not present the typical surface plasmon band in the UV-Vis spectra, which is commonly used for the size characterization of metallic nanoparticles. Total X-ray Reflection Fluorescence (TXRF) was used to determine the amount of elemental gold of the stock solution. This value was found to be $1.03 \mathrm{mg}$ $\mathrm{Au} / \mathrm{mL}$. The concentration of AuNPs was estimated considering that a gold nanoparticle with an average radius of $1.7 \mathrm{~nm}$ may contain $1.79 \times 10^{3}$ gold atoms. Thus, a stock solution of $2.8 \mu \mathrm{M}$ AuNPs was employed.

\section{AA films Electrode modification}

AA films SPCEs modification were carried out as previously described (Revenga-Parra et al. 2012) (a detailed procedure can be found in the supporting information). The modified AA/SPCEs were allow to dry at room temperature and stored protected from light at $4^{\circ} \mathrm{C}$ until use.

\section{ADH electrode modification}

$25 \mathrm{U}$ of Alcohol dehydrogenase were carefully deposited on the AA/SPCE and stored for $1 \mathrm{~h}$ at $4^{\circ} \mathrm{C}$ to assemble the protein on the electrode surface (ADH/AA/SPCE). Finally, the enzyme modified electrode surface was fully covered by $1 \mathrm{mg} / \mathrm{mL}$ chitosan solution in acetic acid solution (3\%) and stored at $4^{\circ} \mathrm{C}$ overnight (CHIT/ ADH/AA/SPCE).

ADH/AuNPs electrode modification. 
$0.5 \mu \mathrm{L}$ of $0.47 \mu \mathrm{M}$ TO-AuNPs were deposited on the AA/SPCE and evaporated to dryness at room temperature (TO-AuNPs/ AA/SPCE). Alcohol dehydrogenase (25U) was carefully deposited on the TO-AuNPs/ AA/SPCE and stored for $1 \mathrm{~h}$ at $4^{\circ} \mathrm{C}$ to assemble the protein on the gold nanoparticles (ADH/TO-AuNPs/ AA/SPCE). Finally, the electrode surface was fully covered by chitosan (CHIT/ ADH/ TO-AuNPs/ AA/SPCE) following the same procedure described above.

For TEM measurements $0.5 \mu \mathrm{L}$ of $1.04 \mu \mathrm{M}$ TO-AuNPs were incubated with alcohol dehydrogenase (25U) for $1 \mathrm{~h}$ at $4^{\circ} \mathrm{C}$. The resulting light brown solution was transferred to a filter unit (Amicon Ultracentrifugal 100K) and filled with water. Samples were centrifuged in a Hettich 320R centrifuge at $4000 \mathrm{rpm}$ for $5 \mathrm{~min}$, this centrifugation process was repeated for 3 times to discard the nanoparticles which were not coordinated into the enzyme structure. Finally, the supernatant was placed on a 400 mesh copper transmission grid (from Lacey Co.) covered with a carbon film and allow to dry at room temperature.

\section{Biosensor response}

Electrocatalytic properties of $\mathrm{ADH}$ biosensor were measuresd by both, cyclic voltammetry (CV) and differential pulse voltammetry (DPV). The CHIT ADH/TOAuNPs/ AA/SPCE was immersed in $1.5 \mathrm{~mL}$ of $0.1 \mathrm{M} \mathrm{pH} 8.0$ PBS containing $1.0 \mathrm{mM}$ $\mathrm{NAD}^{+}$. In CV experiments the potential was seeped from $-0.45 \mathrm{~V}$ to $+0.20 \mathrm{~V}$ at 10.0 $\mathrm{mV} / \mathrm{s}$. DPV was performed fixing the modulation amplitude to $0.05 \mathrm{~V}$ and the modulation time to $0.05 \mathrm{~s}$. The corresponding calibration curves were performed in quiescent solution mode. 


\section{Results and discussion}

ADH biosensor development

Electrode surfaces modified with phenazines and phenothiazines by electrografting can act as adhesives of proteins (Wu et al. 2008, Huang and Wang 2010). Following a similar electrografting strategy, we have modified SPCEs with electroactive films derived from Azure A (AA/SPCE). These films besides to serve as adhesive to immobilize $\mathrm{ADH}$ show a potent and persistent electrocatalytic activity toward the oxidation of NADH. Thus, they can be used as scaffold to build a portable ethanol biosensor in which the enzymaticaliy generated NADH is electrocatalytic oxidized, giving rise to the analytical signal.

Figure 1A shows CV of Azure A films electrografted on SPCEs. The reversible electrochemical process at $-0.25 \mathrm{~V}$ is ascribed to the oxidation and further reduction of the phenothiazine on the electrode surface (curve a). In the presence of $1.0 \mathrm{mM}$ NADH a potent electrocatalytic effect toward NADH oxidation can be observed (curve b). It is noticeable the significant overpotential decrease compared to a bare SPCE (inset Fig. 1A). When these AA/SPCEs are modified with ADH (ADH/AA/SPCEs) a small bioelectrocatalytic current is observed in the presence of $1.0 \mathrm{mM} \mathrm{NAD}^{+}$and $3.0 \mathrm{mM}$ ethanol (Figure 1B). This result suggests that direct immobilization of native ADH on the AA/SPCEs originates changes in the geometry of the active center leading to a poor catalytic response.

Figure1.

Crystallographic studies of native ADH (Hammes-Schiffer and Benkovic 2006) indicates that ethanol is coordinated to the catalytic zinc ion, which is also coordinated with two cysteines and one histidine residue. The $\mathrm{NAD}^{+}$needs to be close to the active center, but without becoming irreversibly entrapped or liked to the enzyme. These structural constraints suggest that any modification in the geometry of the enzyme active site can alter the efficiency of the catalytic process. Although there are many studies describing ethanol biosensors based on $\mathrm{ADH}$, only a few of them clearly demonstrate, by cyclic voltammetry, that the response of the biosensor is based on an EC catalytic process. These biosensors usually involve electrode modification with nanomaterials (Santos et al. 2006, Choi et al. 2007, Liu and Cai 2007, Willner et al. 
2007b, Du et al. 2007). Thus, we have synthesized small size TO-AuNPs. The carboxylic acid functional groups are able to coordinate with the $\mathrm{Zn}$ (II) present in the catalytic center of ADH. Therefore, these functionalized AuNPs represent an attractive ready-to-use tool to promote charge transfer between the immobilized ADH and the electrode surface. HAADF-STEM was used to assess the binding of TO-AuNPs to $\mathrm{ADH}$. In the micrograph shown in Figure 2A it can be observed that the $\mathrm{V}$-shaped molecule matches well with the tridimensional representation (PyMol) of the ADH Type I tetramer (Figure 2C) and along the diffuse domain corresponding to the protein, dense spherical-shaped spots of 3-5 nm are evident. These spots correspond to the gold nanoparticles attached to the tetramer. Figure 2B shows the EDX analysis of one of these spots (white circle in Figure 2A), as can be seen the analysis confirms the presence of gold atoms. In addition, EDX-STEM element mapping of a wider area (white square in Figure 2A) confirms the spacial distribution of the zinc atoms (Figure 2D) and also the gold clusters (Figure 2E) in the ADH.

Figure 2.

Scheme 1.

The as prepared nanoparticles and the ADH were bonded to the AA/SPCE to develop the biosensor (ADH /TO-AuNPs /AA/SPCE), as is depicted in Scheme 1.

The use of bioconjugates enzyme-funtionalized nanoparticle significantly enhances the bioelectrocatalytic response of the biosensor to ethanol (Figure 3A; curve b) compared to that obtained in the absence of AuNPs (Figure 1B; curve b).

Figure 3.

In order to establish if the bioelectrocatalytic current observed is only due to the immobilized enzyme and not also to enzyme molecules that can leak from the biosensing layer, a ADH/TO-AuNPs/ AA/SPCE biosensor was immersed in $1.5 \mathrm{~mL}$ of a solution containing $1.0 \mathrm{mM} \mathrm{NAD}^{+}$and $3.0 \mathrm{mM}$ ethanol in $0.1 \mathrm{M} \mathrm{pH} 8.0 \mathrm{PBS}$. The CV response from $-0.45 \mathrm{~V}$ to $0.2 \mathrm{~V}$ at $10.0 \mathrm{mV} / \mathrm{s}$ was obtained. Then, $100 \mu \mathrm{L}$ of the solution was removed from the electrochemical cell and mixed with $900 \mu \mathrm{L}$ of $0.1 \mathrm{M} \mathrm{pH}$ 8.0 PBS containing $1.0 \mathrm{mM} \mathrm{NAD}{ }^{+}$. The presence of $\mathrm{NADH}$ enzymatically generated was checked recording the UV-Vis absorbance spectrum from 200-800 nm. The first 
absorption peak (Figure 3B) observed is due to the NADH generated during the biosensing electrochemical assay that has diffused to the solution. Successive additions of ethanol result in an increase of the absorption peak, suggesting the presence in solution of active ADH that has leaked from the biosensing layer. To prevent leakage of ADH from the biosensor, its surface was covered with a solution of chitosan (see Experimental section). This polymer forms a stable and hydrophobic barrier avoiding enzyme leakage. Figure 3C depicts the NADH UV-Vis absorption spectra obtained for the chitosan-covered biosensor (CHIT/ADH/TO-AuNPs/AA/SPCE). As in the previous case, it can be observed the absorbance peak due to the NADH generated in the biosensing electrochemical assay. The height of this peak is very similar to that obtained for the biosensor without chitosan, since both experiments are carried out under the same conditions, suggesting that chitosan allows the diffusion of ethanol and $\mathrm{NADH}$ from the solution to the biosensing layer and vice versa. However, in this case the NADH absorbance peak remains constant when increasing amounts of ethanol are added to the solution. This indicates that after covering with chitosan, the ADH is retained on the biosensor surface. Therefore in the subsequent experiments chitosan is included in the biosensor assembly in order to protect and preserve the bio-sensing layer.

On performing analytical determinations much higher sensitivity can be obtained using DPV. Thus, we have employed this technique in the following studies. Figure 4A shows the DPV biosensor response to increasing amounts of ethanol. The typical MichaelisMenten response can be observed by plotting $\mathrm{I}_{\text {cat }} / \mathrm{I}_{0}$ (where $\mathrm{I}_{\text {cat }}$ and $\mathrm{I}_{0}$ are the DPV peak current in the presence and absence of ethanol, respectively) versus ethanol concentration (Figure 4B), suggesting that the analytical signal is controlled by the enzymatic catalysis.

To assess the important role of the AuNPs in the bioelectrocatalytic process, the biosensor was also prepared using $\mathrm{ADH}$ without TO-AuNPs and its response was compared to that obtained with the bioconjugated. As can be observed in Figure 4B, the coordination of $\mathrm{ADH}$ to TO-AuNPs causes a dramatic increase in the biosensor response. As one would expect, in the absence of enzyme the addition of ethanol did not cause any bioelectrocatalytic current.

The apparent Michaelis-Menten constant $\left(\mathrm{K}_{\mathrm{M}}\right)$ calculated from the Lineweaver-Burk equation $\left(1 / \mathrm{I}_{\mathrm{cat}}=1 / \mathrm{I}_{\max }+\mathrm{K}_{\mathrm{M}} / \mathrm{I} \max 1 / \mathrm{C}\right)$ was found to be $0.63 \mathrm{mM}$. This value is lower or similar to that reported for other ADH-dyes modified electrodes (Nasri et al. 2013). 
This result indicates that $\mathrm{ADH}$ retains its great affinity to ethanol after being coordinated to the TO-AuNPs immobilized on AA films.

Figure 4.

\section{Optimization of the biosensor components}

The amount of both $\mathrm{ADH}$ and gold nanoparticles in the biosensor development was optimized. For these porpoise biosensors were prepared using increasing amounts of both components and their responses to ethanol were obtained. The response increases considerably as the units of enzyme deposited on the electrode surface increase from 5 to $25 \mathrm{U}$ and then decreases (Figure 2A SI). This decay suggests that an excess of protein immobilized on the biosensing layer prevents the charge transfer towards the electrode surface (Tsai et al 2007). According with these results 25U of $\mathrm{ADH}$ were chosen as optimal.

As one would expect, the biosensor response shows a significant dependence with the amount of AuNPs present in the biosensing layer. Biosensors were prepared using 0.5 $\mu \mathrm{L}$ of $0.35,0.47,0.70$ and $1.4 \mu \mathrm{M}$ TO-AuNPS solutions, being the response highest for $0.5 \mu \mathrm{L}$ of $0.47 \mu \mathrm{M}$. Higher loadings causes a decrease in the biosensor response, probably due to the steric impediments to enzyme orientation caused by AuNPs electrode saturation.

As mentioned above, $\mathrm{ADH}$ requires the presence of two substrates in the active site: ethanol and $\mathrm{NAD}^{+}$. The effect of $\mathrm{NAD}^{+}$concentration (from 0.2 to $3.0 \mathrm{mM}$ ) on the ethanol biosensor response was evaluated in $0.1 \mathrm{M} \mathrm{pH}$ 8.0 PBS with $1 \mathrm{mM}$ ethanol. The biosensor response increases on increasing $\mathrm{NAD}^{+}$concentration up to $1.0 \mathrm{mM}$ (Figure 2B SI) and then decreases, probably due to inhibition effects by excess of substrate (Utecht 1994).

The mechanism of alcohol oxidation catalyzed by ADH is based on the direct transfer of hydride from the substrate to the cofactor coupled to the transfer of a proton. In addition, the electrocatalytic oxidation of the enzymatically generated NADH (essential for the analytical response of the biosensor) involves two electrons and two protons. Therefore, one would expect a $\mathrm{pH}$ dependent biosensor response. To assess this, the biosensor response was assayed at different $\mathrm{pH}$ from 5.0 to 9.0. The maximum response was obtained at pH 7.0 - 8.0 (Figure 2C SI). Hence, 0.1 M pH 8.0 PBS was selected for ethanol determination. 


\section{Analytical parameters of the biosensor}

The analytical parameters of the ethanol biosensor prepared under optimal conditions were evaluated. The data presented are the average value of three measurements. The calibration curve obtained at $-0.25 \mathrm{~V}$, by plotting $\mathrm{I}_{\text {cat }} / \mathrm{I}_{0}$ versus ethanol concentration depicts a linear behavior up to $2.0 \mathrm{mM}(\mathrm{y}=1.01 \pm 0.02+0.076 \pm 0.001 \mathrm{x} ; \mathrm{r}=0.989)$. The sensitivity (calculated from the slope of the plot) was found to be $0.076 \pm 0.001$ $\mathrm{mM}^{-1}$. The detection and determination limits were calculated as the concentration that gave a signal equal to three and ten times the standard deviation of background current, respectively. Values of $0.14 \pm 0.01$ and $0.36 \pm 0.01 \mu \mathrm{M}$ for the detection and the determination limit were obtained, respectively. These values compare extremely favorably with that obtained for other similar ADH based biosensor reported in the literature (see Table 1). It is worth to note that beside the improved sensitivity the developed device exhibits a highly efficient ethanol oxidation at much lower potential (Gao et al, 2013). Moreover, the reproducibility of the biosensor was evaluated comparing the analytical signals obtained using four different biosensors prepared in the same manner and from the same enzyme batch. A value of $8 \%$, which compares well to the others biosensor, was obtained. Concerning the long-term stability it was found that, if between measurements biosensor is stored at $4{ }^{\circ} \mathrm{C}$, the response remains constant for at least one month. In the light of the results summarized in Table 1 and compared with others ADH based biosensor previously reported in the literature, we concluded that the biosensor developed has a low detection limit and can be used to ethanol determination with good reproducibility in a range appropriate to the quantification of ethanol in blood for a reasonable period of time.

Table1.

\section{Conclusions}

Nanometric size AuNPs capped with thiotic acid have been synthesized to coordinate with the $\mathrm{Zn}$ (II) present in the catalytic center of $\mathrm{ADH}$ dehydrogenase (ADH). TEM images confirm that the functionalized gold nanoparticles are included in the ADH. Combined to electrogenerated Azure A films they have been used for ethanol biosensors. The presence of the functionalized AuNPS enhances up to 10 times the 
bioelectrocatalytic response of the as prepared ethanol biosensor, which show low potential response of $-0.25 \mathrm{~V}$ besides a very good analytical performance.

\section{Acknowledgements.}

This work has been supported by the Comunidad Autonoma de Madrid (NANOAVANSENS S2013/MIT 3029) and the Spanish Ministerio de Ciencia e Innovacion (project No. CTQ2011-28157). M. P. acknowledges the 2009 Ramon y Cajal program from the Spanish MINECO. 


\section{References.}

Abad, J.M., Gass, M., Bleloch, A., Schiffrin, D.J., 2009. Direct Electron Transfer to a Metalloenzyme Redox Center Coordinated to a Monolayer-Protected Cluster. Journal of the American Chemical Society 131(29), 10229-10236.

Abad, J.M., Mertens, S.F.L., Pita, M., Fernández, V.M., Schiffrin, D.J., 2005. Functionalization of Thioctic Acid-Capped Gold Nanoparticles for Specific Immobilization of Histidine-Tagged Proteins. Journal of the American Chemical Society 127(15), 5689-5694.

Banks, C.E., Compton, R.G., 2005. Exploring the electrocatalytic sites of carbon nanotubes for NADH detection: an edge plane pyrolytic graphite electrode study. Analyst 130(9), 1232-1239.

Banks, C.E., Davies, T.J., Wildgoose, G.G., Compton, R.G., 2005. Electrocatalysis at graphite and carbon nanotube modified electrodes: edge-plane sites and tube ends are the reactive sites. Chemical Communications(7), 829-841.

Bartlett, P.N., Simon, E., Toh, C.S., 2002. Modified electrodes for NADH oxidation and dehydrogenase-based biosensors. Bioelectrochemistry 56(1-2), 117-122.

Barzana, E., Klibanov, A.M., Karel, M., 1989. A colorimetric method for the enzymatic analysis of gases: The determination of ethanol and formaldehyde vapors using solid alcohol oxidase. Analytical Biochemistry 182(1), 109-115.

Brust, M., Walker, M., Bethell, D., Schiffrin, D.J., Whyman, R., 1994. Synthesis of thiol-derivatised gold nanoparticles in a two-phase Liquid-Liquid system. Journal of the Chemical Society, Chemical Communications(7), 801-802.

Cai, C.-X., Xue, K.-H., Zhou, Y.-M., Yang, H., 1997. Amperometric biosensor for ethanol based on immobilization of alcohol dehydrogenase on a nickel hexacyanoferrate modified microband gold electrode. Talanta 44(3), 339-347.

Choi, H.N., Lyu, Y.-K., Han, J.H., Lee, W.-Y., 2007. Amperometric Ethanol Biosensor Based on Carbon Nanotubes Dispersed in Sol-Gel-Derived Titania-Nafion Composite Film. Electroanalysis 19(14), 1524-1530.

Du, P., Liu, S., Wu, P., Cai, C., 2007. Single-walled carbon nanotubes functionalized with poly(nile blue A) and their application to dehydrogenase-based biosensors. Electrochimica Acta 53(4), 1811-1823.

Gao, W., Chen, Y., Xi, J., Lin, S., Chen, Y., Lin, Y., Chen, Z., 2013. A novel electrochemiluminiscence ethanol biosensor based on this(2,2'-bipyridine) ruthenium 
(II) and alcohol dehydrogenase immobilized in graphene/bovine serum albumin composite film. Biosensors \& Bioelectronics 41, 776-782.

García Mullor, S., Sánchez-Cabezudo, M., Miranda Ordieres, A.J., López Ruiz, B., 1996. Alcohol biosensor based on alcohol dehydrogenase and Meldola Blue immobilized into a carbon paste electrode. Talanta 43(5), 779-784.

Goswami, P., Chinnadayyala, S., Chakraborty, M., Kumar, A., Kakoti, A., 2013. An overview on alcohol oxidases and their potential applications. Appl Microbiol Biotechnol 97(10), 4259-4275.

Gutierrez-Sanchez, C.; Pita, M., Vaz-Dominguez, C., Shleev, S., De Lacey, A.L., 2012. Gold nanoparticles as electronic bridges for laccase-based biocathodes. J Am Chem Soc 134, 17212-17220.

Hammes-Schiffer, S., Benkovic, S.J., 2006. Relating Protein Motion to Catalysis. Annual Review of Biochemistry 75(1), 519-541.

Hua, E., Wang, L., Jing, X., Chen, C., Xie, G., 2013. One-step fabrication of integrated disposable biosensor based on $\mathrm{ADH} / \mathrm{NAD}+/$ meldola's blue/graphitized mesoporous carbons/chitosan nanobiocomposite for ethanol detection. Talanta 111(0), 163-169 Huang, H.Y., Wang, C.M., 2010. Phenothiazine: An Effective Molecular Adhesive for Protein Immobilization. The Journal of Physical Chemistry C 114(8), 3560-3567.

Jena, B.K., Raj, C.R., 2006. Electrochemical Biosensor Based on Integrated Assembly of Dehydrogenase Enzymes and Gold Nanoparticles. Analytical Chemistry 78(18), 6332-6339.

Jensen, P.S., Chi, Q., Grumsen, F.B., Abad, J.M., Horsewell, A., Schiffrin, D.J., Ulstrup, J., 2007. Gold Nanoparticle Assisted Assembly of a Heme Protein for Enhancement of Long-Range Interfacial Electron Transfer. The Journal of Physical Chemistry C 111(16), 6124-6132.

Jia, J., Wang, B., Wu, A., Cheng, G., Li, Z., Dong, S., 2002. A Method to Construct a Third-Generation Horseradish Peroxidase Biosensor: Self-Assembling Gold Nanoparticles to Three-Dimensional Sol-Gel Network. Analytical Chemistry 74(9), 2217-2223.

Katz, E., Willner, I., 2004. Integrated Nanoparticle-Biomolecule Hybrid Systems: Synthesis, Properties, and Applications. Angewandte Chemie International Edition 43(45), 6042-6108.

Kowalewska, B., Kulesza, P.J., 2012. Toward More Efficient Bioelectrocatalytic Oxidation of Ethanol for Amperometric Sensing and Biofuel Cell Technology. 
Analytical Chemistry 84(21), 9564-9571

Lazarova, G., Genova, L., Kostov, V., 1987. Ethanol concentration determination using solvent extraction and refractometry. Acta Biotechnologica 7(1), 97-99.

Lee, C.-A., Tsai, Y.-C., 2009. Preparation of multiwalled carbon nanotube-chitosanalcohol dehydrogenase nanobiocomposite for amperometric detection of ethanol. Sensors and Actuators B: Chemical 138(2), 518-523.

Li, L., Lu, H., Deng, L., 2013a. A sensitive NADH and ethanol biosensor based on graphene-Au nanorods nanocomposites. Talanta 113(0), 1-6.

Li, Z., Huang, Y., Chen, L., Qin, X., Huang, Z., Zhou, Y., Meng, Y., Li, J., Huang, S., Liu, Y., Wang, W., Xie, Q., Yao, S., 2013b. Amperometric biosensor for NADH and ethanol based on electroreduced graphene oxide-polythionine nanocomposite film. Sensors and Actuators B: Chemical 181(0), 280-287.

Li, W.J., Wei, X.G., Cong, W., Ouyang, F., 2000. Simultaneous analysis of low concentrations of glucose, ethanol and glycerol by high performance liquid chromatographic method. Se Pu 18(2), 170-172.

Liu, S., Cai, C., 2007. Immobilization and characterization of alcohol dehydrogenase on single-walled carbon nanotubes and its application in sensing ethanol. Journal of Electroanalytical Chemistry 602(1), 103-114.

Nasri, Z., Shams, E., Ahmadi, M., 2013. Direct Modification of a Glassy Carbon Electrode with Toluidine Blue Diazonium Salt: Application to NADH Determination and Biosensing of Ethanol. Electroanalysis 25(8), 1917-1925.

Niemeyer, C.M., 2001. Nanoparticles, Proteins, and Nucleic Acids: Biotechnology Meets Materials Science. Angewandte Chemie International Edition 40(22), 4128-4158. Pariente, F., Lorenzo, E., Tobalina, F., Abruna, H.D., 1995. Aldehyde Biosensor Based on the Determination of NADH Enzymically Generated by Aldehyde Dehydrogenase. Analytical Chemistry 67(21), 3936-3944.

Ragazzo-Sanchez, J.A., Chalier, P., Ghommidh, C., 2005. Coupling gas chromatography and electronic nose for dehydration and desalcoholization of alcoholized beverages: Application to off-flavour detection in wine. Sensors and Actuators B: Chemical 106(1), 253-257.

Raj, S.B., Ramaswamy, S., Plapp, B.V., 2014. Yeast Alcohol Dehydrogenase Structure and Catalysis. Biochemistry 53(36), 5791-5803.

Revenga-Parra, M., Gómez-Anquela, C., García-Mendiola, T., Gonzalez, E., Pariente, F., Lorenzo, E., 2012. Grafted Azure A modified electrodes as disposable $\beta$ - 
nicotinamide adenine dinucleotide sensors. Analytica Chimica Acta 747(0), 84-91.

Rubach, J.K., Plapp, B.V., 2003. Amino Acid Residues in the Nicotinamide Binding Site Contribute to Catalysis by Horse Liver Alcohol Dehydrogenase Biochemistry 42(10), 2907-2915.

Rubianes, M.D., Rivas, G.A., 2005. Enzymatic Biosensors Based on Carbon Nanotubes Paste Electrodes. Electroanalysis 17(1), 73-78.

Santos, A.S., Pereira, A.C., Durán, N., Kubota, L.T., 2006. Amperometric biosensor for ethanol based on co-immobilization of alcohol dehydrogenase and Meldola's Blue on multi-wall carbon nanotube. Electrochimica Acta 52(1), 215-220.

Scott, D., Toney, M., Muzikár, M., 2008. Harnessing the Mechanism of Glutathione Reductase for Synthesis of Active Site Bound Metallic Nanoparticles and Electrical Connection to Electrodes. Journal of the American Chemical Society 130(3), 865-874.

Serafín, V., Agüí, L., Yáñez-Sedeño, P., Pingarrón, J.M., 2011. A novel hybrid platform for the preparation of disposable enzyme biosensors based on poly(3,4ethylenedioxythiophene) electrodeposition in an ionic liquid medium onto gold nanoparticles-modified screen-printed electrodes. Journal of Electroanalytical Chemistry 656(1-2), 152-158.

Shan, C., Yang, H., Han, D., Zhang, Q., Ivaska, A., Niu, L., 2010. Electrochemical determination of $\mathrm{NADH}$ and ethanol based on ionic liquid-functionalized graphene. Biosensors \& Bioelectronics 25(6), 1504-1508

Tian, J., Deng, S.-Y., Li, D.-L., Shan, D., He, W., Zhang, X.-J., Shi, Y., 2013. Bioinspired polydopamine as the scaffold for the active AuNPs anchoring and the chemical simultaneously reduced graphene oxide: Characterization and the enhanced biosensing application. Biosensors \& Bioelectronics 49(0), 466-471.

Tkac, J., Svitel, J., Vostiar, I., Navratil, M., Gemeiner, P., 2009. Membrane-bound dehydrogenases from Gluconobacter sp.: Interfacial electrochemistry and direct bioelectrocatalysis. Bioelectrochemistry 76(1-2), 53-62.

Tsai, Y.C.; Huang, J.D.; Chiu, C.C, 2007. Amperometric ethanol biosensor based on poly(vinyl alcohol)-multiwalled carbon nanotube-alcohol dehydrogenase biocomposite. Biosensors \& Bioelectronics 22, 3051-3056

Utecht, R.E., 1994. A Kinetic Study of Yeast Alcohol Dehydrogenase. Journal of Chemical Education 71(5), 436.

Wanekaya, A.K., Uematsu, M., Breimer, M., Sadik, O.A., 2005. Multicomponent analysis of alcohol vapors using integrated gas chromatography with sensor arrays. 
Sensors and Actuators B: Chemical 110(1), 41-48.

Willner, I., Baron, R., Willner, B., 2007a. Integrated nanoparticle-biomolecule systems for biosensing and bioelectronics. Biosensors \& Bioelectronics 22(9-10), 1841-1852.

Willner, I., Basnar, B., Willner, B., 2007b. Nanoparticle-enzyme hybrid systems for nanobiotechnology. FEBS Journal 274(2), 302-309.

Wiseman, A., 1988. Ethanol detection and assay systems: Development of immobilised enzyme biosensors. TrAC Trends in Analytical Chemistry 7(1), 5-7.

Wu, S.-W., Huang, H.Y., Guo, Y.C., Wang, C.M., 2008. ALO-Patternable Artificial Flavin: Phenazine, Phenothiazine, and Phenoxazine. The Journal of Physical Chemistry C 112(25), 9370-9376.

Xiao, Y., Patolsky, F., Katz, E., Hainfeld, J.F., Willner, I., 2003. "Plugging into Enzymes": Nanowiring of Redox Enzymes by a Gold Nanoparticle. Science 299(5614), 1877-1881.

Zhao, J., O'Daly, J.P., Henkens, R.W., Stonehuerner, J., Crumbliss, A.L., 1996. A xanthine oxidase/colloidal gold enzyme electrode for amperometric biosensor applications. Biosensors and Bioelectronics 11(5), 493-502.

Zhen, S., Wang, Y., Liu, C., Xie, G., Zou, C., Zheng, J., Zhu, Y., 2011. A novel microassay for measuring blood alcohol concentration using a disposable biosensor strip. Forensic Science International 207(1-3), 177-182. 


\section{Figure captions}

Fig.1. A) Cyclic voltammograms of an AA/SPCE or SPCE (Inset) in $0.1 \mathrm{M}$ pH $8.0 \mathrm{PBS}$ before (curve a) and after (curve b) addition of $1.0 \mathrm{mM}$ NADH. B) Cyclic voltammograms of an AA/SPCE modified with $\mathrm{ADH}$, in $0.1 \mathrm{M} \mathrm{pH}$ 8.0 PBS solution containing $1.0 \mathrm{mM}$ of $\mathrm{NAD}^{+}$before (curve a) and after (curve b) addition of $3.0 \mathrm{mM}$ ethanol.

Figure 2. A) HAADF-STEM image of ADH after incubation with TO-AuNPs. B) EDX spectra of the white circle area of figure A. C) Structure of Alcohol Dehydrogenase Type I from Saccaromyces Cerevisiae. D) and E) EDX-TEM element mapping of the white squared area of figure A for zinc (figure D) and gold (figure E).

Scheme 1. Scheme depicting the processes for the biosensor development.

Fig.3. A) Cyclic voltammogram of an ADH/TO-AuNPs/AA/SPCE in 0.1M pH 8.0 PBS containing $1.0 \mathrm{mM}$ of NAD ${ }^{+}$before (curve a) and after (curve b) the addition of $3.0 \mathrm{mM}$ ethanol. UV-Vis absorption spectra of an aliquot of the solution used in figure A after addition of increasing amounts of ethanol when ADH/TO-AuNPs/AA/SPCE (B) or CHIT/ADH/TO-AuNPs/AA/SPCE (C) biosensors were employed.

Fig.4. A) Differential pulse Voltammograms of a CHIT/ADH/TO-AuNPs/AA/SPCE in $0.1 \mathrm{M} \mathrm{pH}$ 8.0 PBS containing $1.0 \mathrm{mM}$ of $\mathrm{NAD}^{+}$for successive additions of ethanol. B) Biosensor current response $\left(\mathrm{I}_{\mathrm{cat}} / \mathrm{I}_{0}\right)$ to ethanol in the presence (squares) or absence (triangles) of AuNPs at the same conditions as in figure A. 
Table1. Comparison between the analytical parameters of the proposed method and other reported ADH biosensors.

\begin{tabular}{lcccc}
\hline \multicolumn{1}{c}{ Biosensor } & LOD $(\boldsymbol{\mu M})$ & Linear range $(\mathbf{m M})$ & $\boldsymbol{R S D}(\%)$ & Ref \\
\hline \hline $\begin{array}{l}\text { AuNPs/ADH-MPTS/Au } \\
\text { ADH/AuNPs/PDRGO/GE }\end{array}$ & 20 & $0.5-2.5$ & n.d. & (Jena and Raj 2006) \\
ADH/PDDA-CNTs/GCE & 24 & $0.1-3.0$ & 7.8 & $\begin{array}{c}\text { (Tian et al.2013) } \\
\text { (Kowalewska and } \\
\text { Kulesza 2012) }\end{array}$ \\
ADH/IL-graphene/Chit/GCE & 5.0 & $0.05-0.3$ & 3.1 & (Shan et al.2010) \\
ADH/PEDOT/AuNPS/SPCE & 2.0 & $0.025-0.2$ & 4.2 & (Serafin et al 2011) \\
ADH/GO/Ru(bpy) 3 /GCE & 0.1 & $0.005-0.1$ & 4.4 & (Gao et al.2013 \\
ADH-/ MDB/GMCs/SPCE & 80 & $0.001-2.0$ & 9.7 & (Hua et al.2013) \\
ADH/ERGO-PTH/GCE & 0.3 & $0.5-15$ & 3.8 & \\
ADH/TB/GCE & 140 & $0.05-1.0$ & 5.2 & (Li et al 2013b) \\
ADH/GN-AuNrs/GCE & 1.5 & $0.5-4.0$ & $<6$ & (Nasri et al.2013) \\
Chit/ADH-AuNPs/AA/SPCE & 0.14 & $0.005-0.4$ & 4.6 & (Li et al.2013a) \\
\hline
\end{tabular}



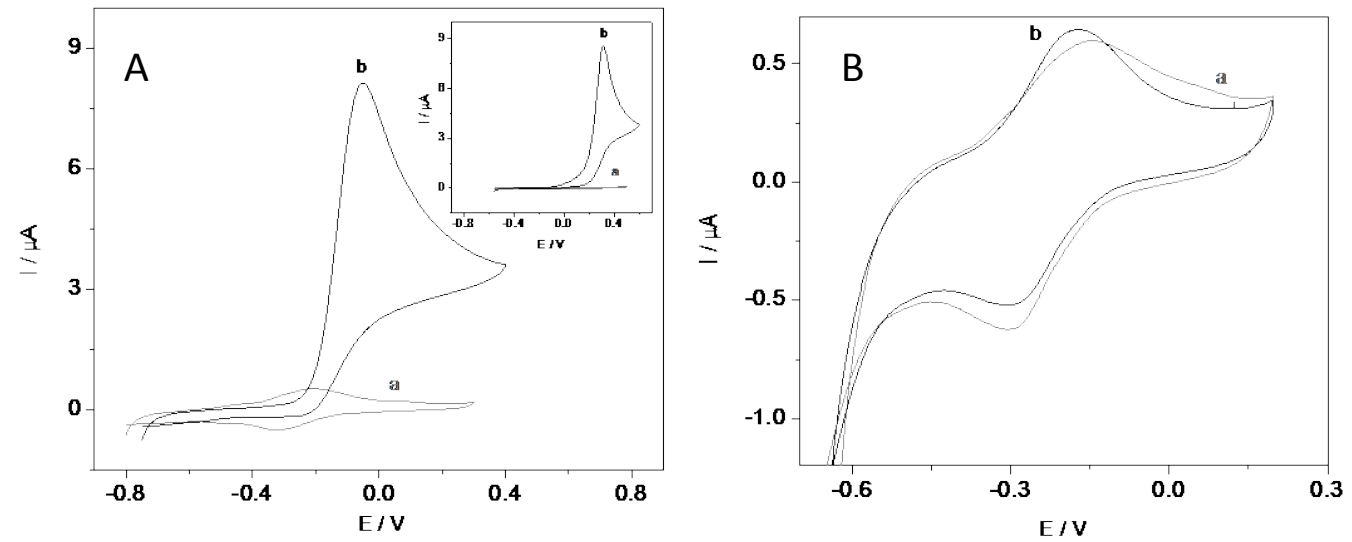

Figure1.
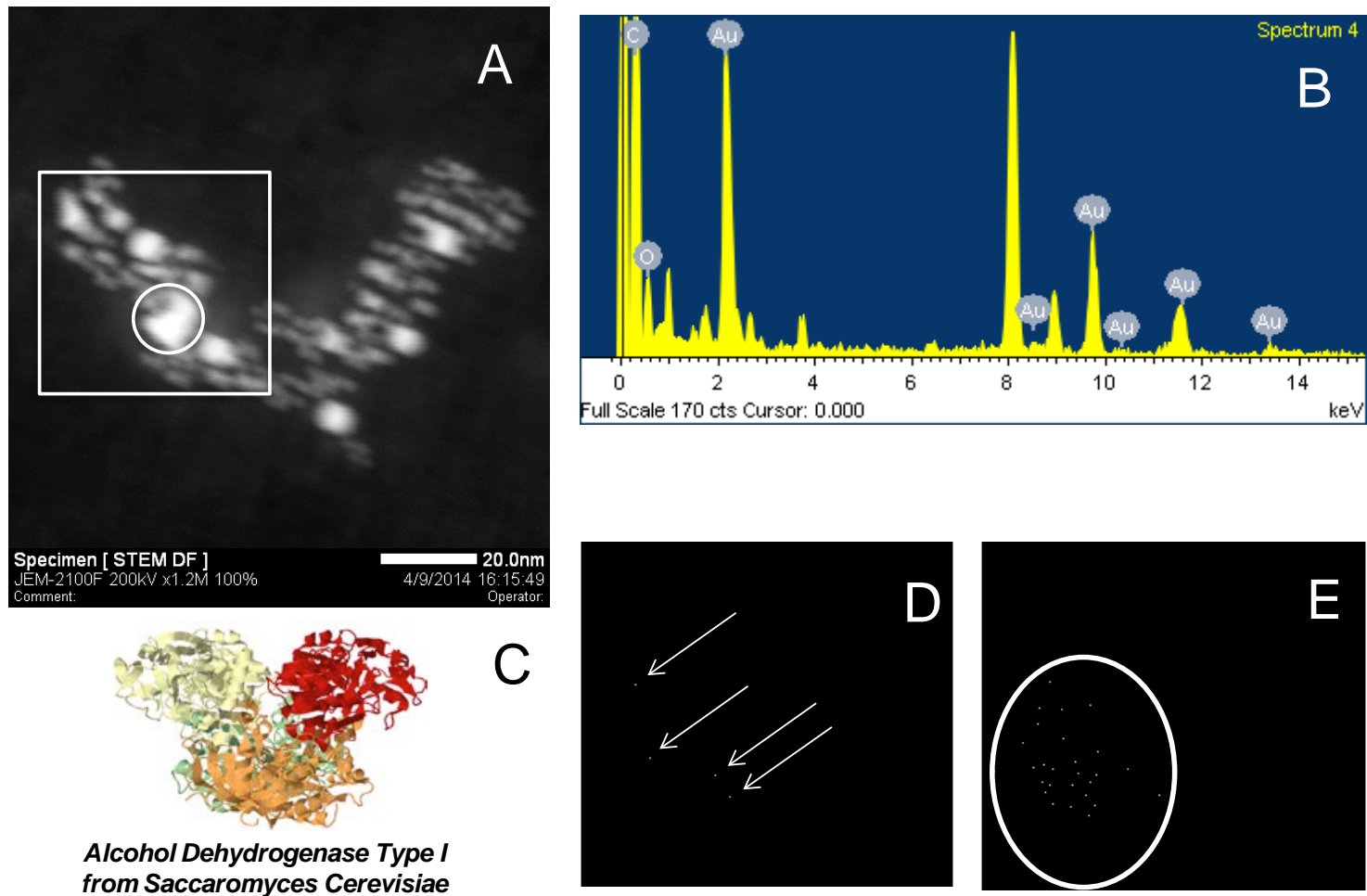

Figure 2. 


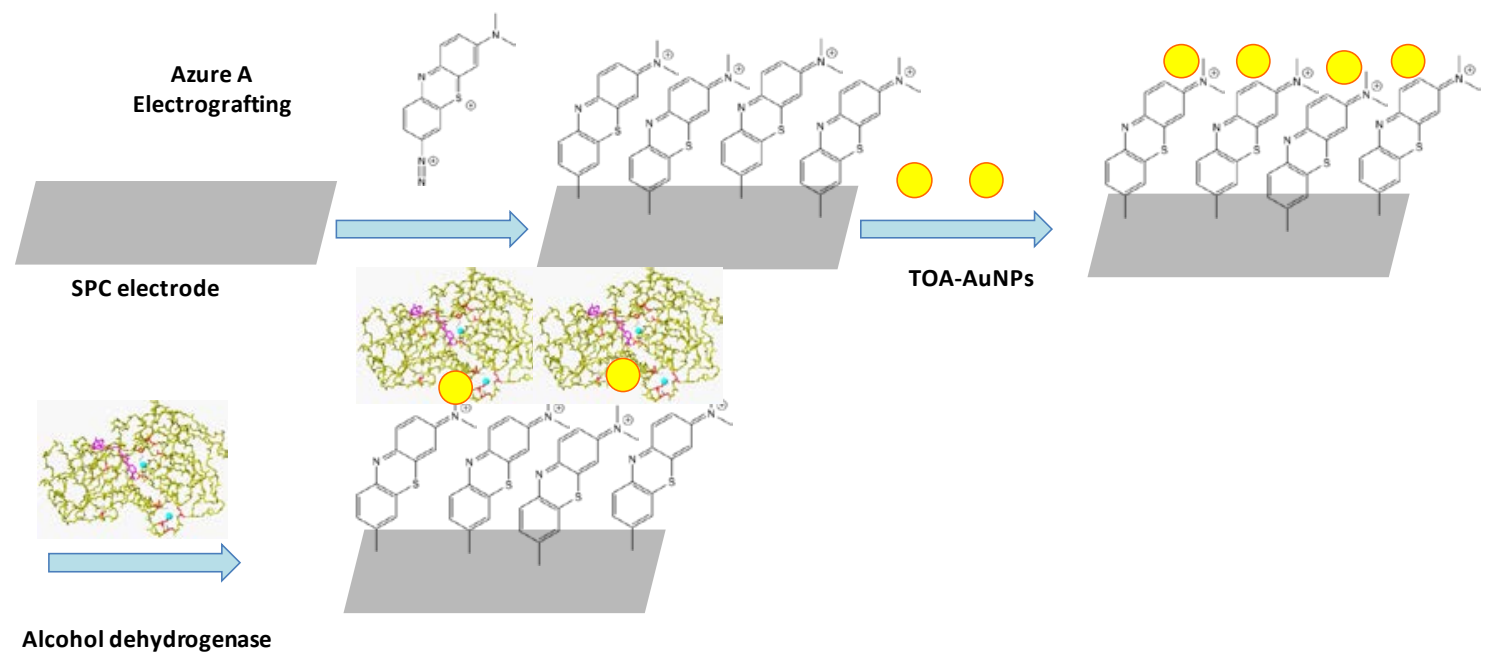

Scheme 1.
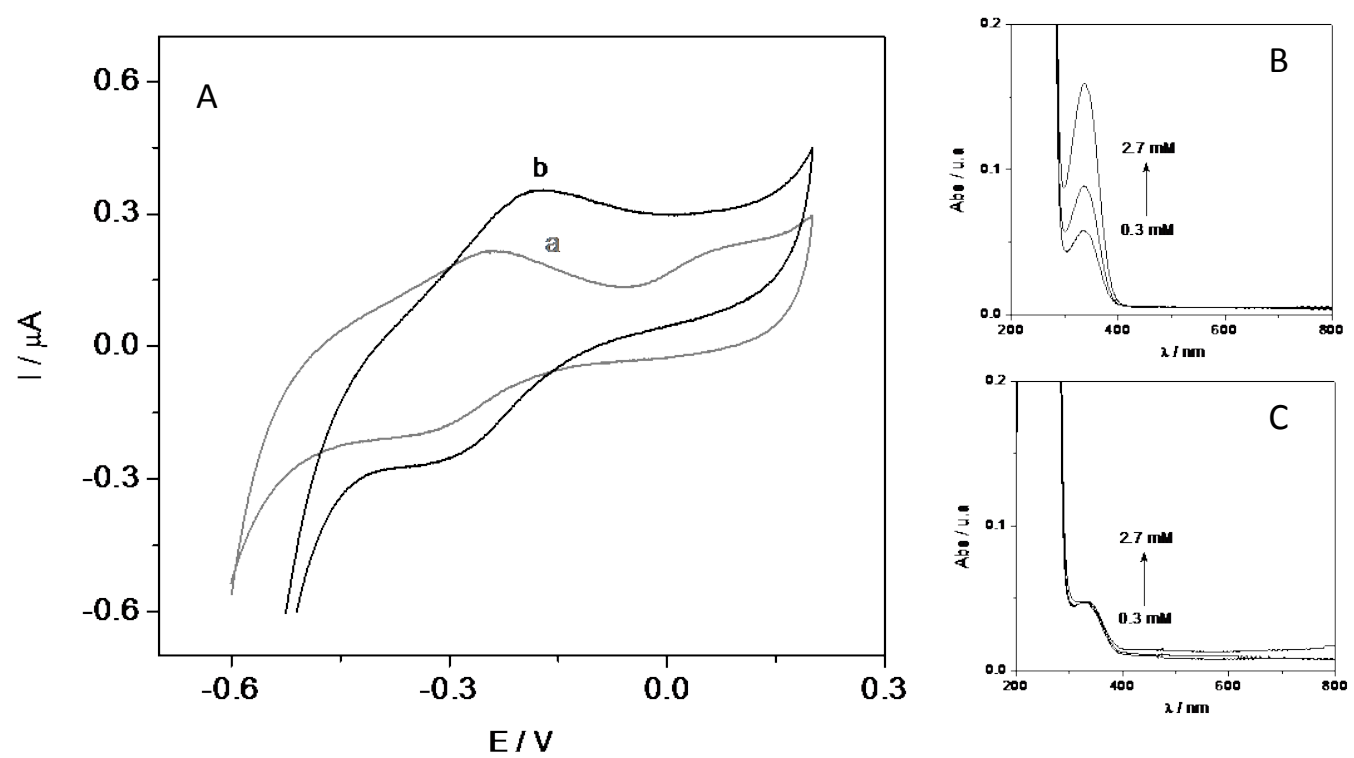

Figure 3. 

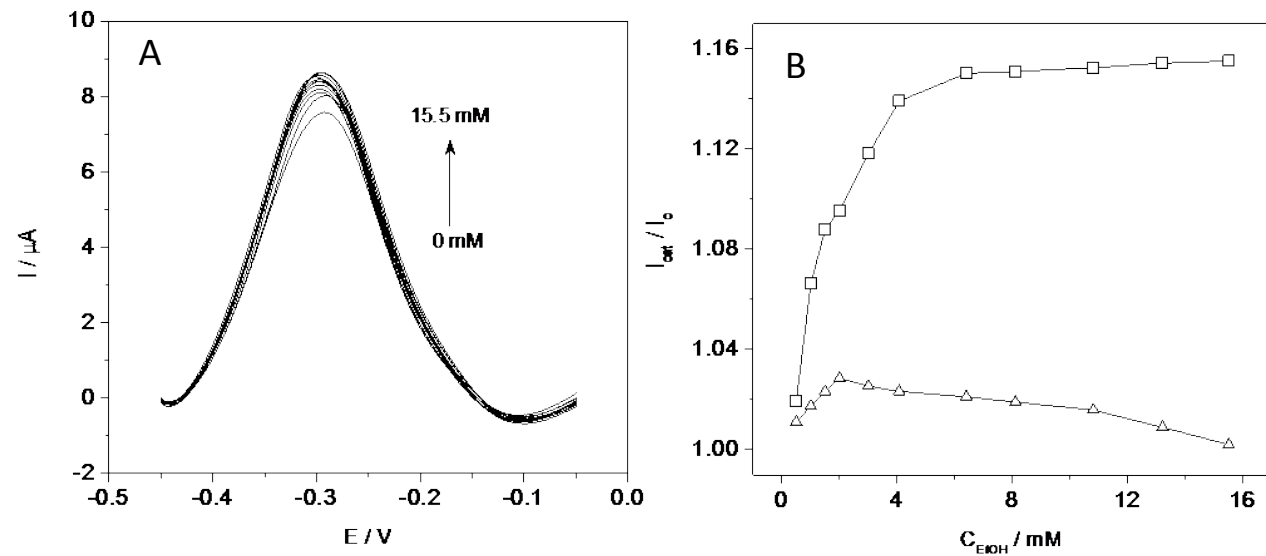

Figure 4. 